\title{
Consideration of Uneven Misclassification Cost and Group Size for Bankruptcy Prediction
}

\author{
Yi-Chun Kuo \\ Department of International Business, Chung Yuan Christian University, Chungli, Taiwan. \\ Email: chun@cycu.edu.tw \\ Received October $19^{\text {th }}, 2013$; revised November $19^{\text {th }}$, 2013; accepted December $6^{\text {th }}, 2013$ \\ Copyright (C) 2013 Yi-Chun Kuo. This is an open access article distributed under the Creative Commons Attribution License, which \\ permits unrestricted use, distribution, and reproduction in any medium, provided the original work is properly cited. In accordance of \\ the Creative Commons Attribution License all Copyrights (C) 2013 are reserved for SCIRP and the owner of the intellectual property \\ Yi-Chun Kuo. All Copyright (C) 2013 are guarded by law and by SCIRP as a guardian.
}

\begin{abstract}
Despite a larger number of approaches developed for predicting bankruptcy over the past three decades, rare research has considered the effects of misclassification cost and group size. Uneven cost of misclassification results from Type I (misclassify a healthy company as a failure) and Type II errors (misclassify a failed company as healthy), which are seldom considered. Without accounting for unevenness in misclassification cost, the classifier is developed based on minimizing total misclassification errors to improve the hit-ratio for classification performance. This not only results in poor decision capability, but also causes bias towards the larger group. This paper explores the issues of uneven misclassification costs and imbalanced group size by applying an asymmetric-stratified data envelopment analysis to bankruptcy prediction. The results show a tradeoff between hit-ratio and misclassification cost when Type II error cost is ten times over that of Type I, that is, the higher the hit-ratio is, the greater the resulting misclassification costs are. By incorporating different proportions of Type II error costs to Type I into the classification procedures, the proposed approach provides greater flexibility to decision makers for credit evaluation or bankruptcy prediction based on different risk attitudes and situations.
\end{abstract}

Keywords: Bankruptcy Prediction; Misclassification Cost; Data Envelopment Analysis (DEA)

\section{Introduction}

Decision-making problems in the area of bankruptcy prediction, credit evaluation, and its risk measurement have been considered extremely important but difficult tasks for financial institutions due to the high level of risk from wrong decisions. A wrong credit decision results in refusing good credit, which causes loss of future profit margins (commercial risk), or approving bad credit, which causes loss of interest and principal money (credit risk). The same risk exists for bankruptcy prediction from the misclassification of a failed company as a healthy one. The academic and business community has long regarded the development of a bankruptcy predicttion model as an important issue that has been widely studied. Several review articles have investigated and compared many useful techniques for bankruptcy prediction. Altman [1] proposed discriminant analysis for the prediction of business failure risk, in which bankruptcy could be explained using a combination of five (selected from an original list of 22) financial ratios. Subsequently, the use of this method spread to discriminant models of predicting business failure [2,3]. However, conventional statistical methods have some restrictive assumptions such as linearity, normality, and independence among predictor variables. But the violation of these assumptions for independent variables frequently occurs with financial data [4].

Several recent studies have applied data envelopment analysis (DEA) for the classification problem [5-13] Troutt et al. [5] revealed important features of DEA models for potential use in classification, for example, 1) the capability to manage nonparametric data; 2) the capability to develop frontiers when information about only one class is available; 3 ) the assumption about class convexity; 4) the piecewise nonlinear classification boundary; 5) the capability to solve inverse classification problems; and 6) a single classification boundary for a given training data. The aforementioned specialties make DEA a 
valid approach to bankruptcy prediction, but the assumption of no Type II errors in developing the acceptance boundary of credit applicants in the Troutt study might restrict the risk attitude or flexibility of manager judgment in practice.

This paper proposes a new approach called the asymmetric-stratified DEA for predicting business failure. Two piecewise boundaries of non-bankrupt and bankrupt groups established by the benchmarks of both groups are used as separation functions. The idea for this approach was inspired by the fact that firms belonging to the same group should be dominated by the same benchmarks; thus, such benchmarks can be used to construct the group boundary. The benchmarks of non-bankrupt and bankruptcy group are identified based on opposite viewpoints. The non-bankrupt group identifies the worst firms as benchmarks based on how efficient they are at being bad, and firms dominated by such benchmarks are evaluated as inefficient and regarded safer than benchmarks that become bankrupt. In contrast, the boundary of the bankruptcy group is established by picking out the best companies as benchmarks based on how efficient they are at being good. Firms dominated by both boundaries represent the existing overlap. The risk of Type I (misclassifying a healthy firm as a failure) and Type II errors (misclassifying a failed company as healthy) may occur. Here, an asymmetric-stratified DEA model with a layering technique is applied to eliminate the overlap to establish separating hyperplanes. The major merit of the proposed approach is its ability to establish nonlinear separating hyperplanes easily by the benchmarks of the two groups without needing to pre-specify the classification function as the parametric methods do. By incorporating the risk and cost of Type I and Type II errors into the layering DEA procedure, the classification functions are determined through minimizing misclassification cost, typically ignored in certain approaches using hit-ratio as the indicator of correct classification. Particularly in an uneven group case (the population of bankrupt and nonbankrupt companies is commonly uneven, [14]), the rule of most approaches tends to have upward bias toward the larger group (the non-bankrupt group) to increase the hitratio. The proposed approach is more practicable for the case of uneven misclassification costs and imbalanced group size.

\section{Methodology}

Charnes et al. [15] first introduced DEA. Consider $\mathrm{n}$ production units or decision-making units (DMUs) to be evaluated using the same $\mathrm{m}$ inputs to produce $\mathrm{s}$ different outputs. Let $X_{i}$ be the input consumption vector from $D M U_{i}$ with $X_{i}=\left(x_{1 i}, \cdots, x_{m i}\right)^{\mathrm{T}}$, and $Y_{i_{\mathrm{T}}}$ the output production vector, where $Y_{i}=\left(y_{1 i}, \cdots, y_{s i}\right)^{\mathrm{T}}$. The DEA input-oriented efficiency score $\theta$ is given by

The DEA model classifies DMUs on the frontier as efficient and DMUs enveloped by the frontier as inefficient. Thus, the benchmarks are the best performers on the frontier, and the poor performers are furthest away from the frontier. Instead of picking out good performers, this article establishes the frontier of the non-bankrupt group by identifying the worst performers to be benchmarks. This is achieved by selecting variables that reflect bad performance. The strategy is to choose output variables that reflect poor utilization of resources, or undesirable outcomes, such as working capital and debt. For input variables, profits, sales, and equity (marked as $Z_{1}$ and $Z_{2}$ in Figure 1(a)) are selected which are the less the better for a bad performer. The companies identified to construct the frontier of the non-bankrupt group are those companies (shown as points A, B, C in Figure 1(a)) with the lowest inputs (profits, sales, et al. ).

$$
\begin{array}{ll} 
& \min _{\theta, \lambda} \theta \\
\text { s.t. } & \sum_{i=1}^{n} \lambda_{i} X_{i} \leq \theta X^{\prime} \\
& \sum_{i=1}^{n} \lambda_{i} Y_{i} \geq Y^{\prime} \\
& \lambda_{i} \geq 0, \quad i=1, \cdots, n
\end{array}
$$

The frontier of the bankrupt group is established based on a general DEA model to identify the best performers with the highest level of outputs (shown as points D, E, and $\mathrm{F}$ in Figure 1(b), here $Z_{1}$ and $Z_{2}$ are defined as output variables). The variables used to identify the frontiers of non-bankrupt and bankrupt groups need to be the same, that is, one variable used as output for non-bankruptcy will be applied as input for the bankrupt group and vice versa. The major reason is that DUMs identified as the benchmarks of each group, will be used as variablebenchmarks [16] to evaluate all companies, including non-bankruptcy (notated as $G_{1}$ ) and bankruptcy (notated as $G_{2}$ ) to classify their membership based on the same measurements.

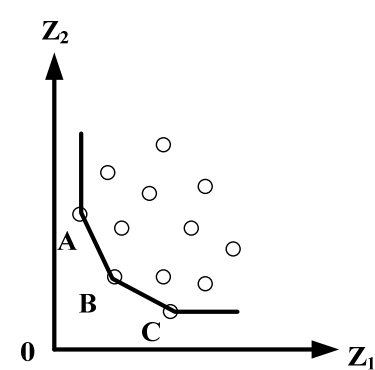

(a)

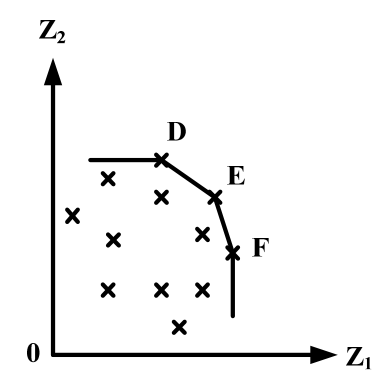

(b)
Figure 1. Frontiers with worse practices in (a) and best practices in (b). 
Define $J_{1}=\left\{D M U_{j}, j=1,2, \cdots, n_{1}\right\}$ for all the DMUs of $G_{1}$ (The same definition for $G_{2}$ ). The set $E^{*}$ represents the benchmarks identified by model (1) where $E^{*}=\left\{D M U_{k} \in J_{1} \mid \theta_{k}=1\right\}$. If $E^{*}$ of one group is used as the benchmark to evaluate all DMUs, $E^{*}$ is referred to as the variable-benchmark. The variable benchmark model for $G_{1}$ is formulated below: (The formula is the same for $\left.G_{2}\right)$

$$
\begin{array}{ll}
\min & \delta_{G_{1}} \\
\text { s.t. } & \sum_{i \in E^{*}} \lambda_{i} x_{p i} \leq \delta_{G_{1}} x_{p}^{\text {new }} \\
& \sum_{i \in E^{*}} \lambda_{i} y_{r i} \geq y_{r}^{\text {new }} \\
& \lambda_{i} \geq 0, \quad i \in E^{*}
\end{array}
$$

The efficiency score $\delta$ is expressed as a number between 0 - 1. A $D M U^{\text {new }}$ with a score less than one is deemed inefficient relative to other DMUs. A company evaluated to be inefficient by the variable-benchmarks of one frontier, is dominated by such a frontier. Those companies dominated by the same frontier are in the same production possibility set (PPS) and classified to the same group. Two PPSs of $G_{1}$ and $G_{2}$ might have an intersection $\left(P P S_{1}^{1} \cap P P S_{2}^{1}\right)$ (shown as the shadow area in Figure 2), which means some companies or DMUs are dominated by the frontiers of $G_{1}$ and $G_{2}$ simultaneously, which may result in misclassification. That is, if $\delta_{G_{1}}$ and $\delta_{G_{2}}$ are the efficiency scores of $D M U^{\text {new }}$ evaluated separately by the variable-benchmarks of $G_{1}$ and $G_{2}$, there will be four possible situations:

$$
\begin{array}{ll}
\text { (i). } & \delta_{G_{1}} \leq 1, \quad \delta_{G_{2}}>1 \\
\text { (ii). } & \delta_{G_{1}}>1, \quad \delta_{G_{2}} \leq 1 \\
\text { (iii). } & \delta_{G_{1}}<1, \quad \delta_{G_{2}}<1 \\
\text { (iv). } & \delta_{G_{1}}>1, \quad \delta_{G_{2}}>1
\end{array}
$$

Situation (iii) indicates the intersection between two PPSs because $D M U^{\text {new }}$ is dominated by the benchmarks of $G_{1}$ and $G_{2}$ simultaneously. To establish a general discriminant rule that a company can be classified to a des-

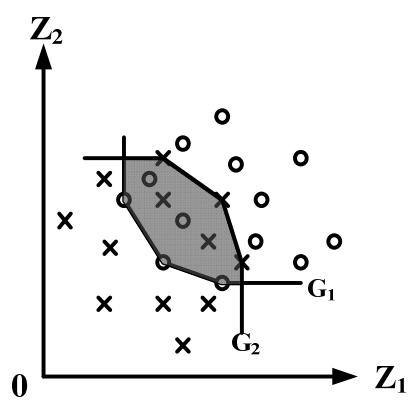

Figure 2. Intersection (the shadow area) between the two groups. ignated group if it is dominated only by a correspondent frontier, the stratification model [16] is further applied to deal with the overlap problem.

Define $J^{l}=\left\{D M U_{j}, j=1,2, \cdots, n_{1}\right\}$ for all the DMUs of $G_{1}$ (The same definition and process are for $\left.G_{2}\right) . \quad J^{l+1}=J^{l}-E^{l}$, where

$E^{l}=\left\{D M U_{k} \in J^{l} \mid \theta^{*}(l, k)=1\right\}$ and $\theta^{*}(l, k)$ is the optimal value of the following model when $\mathrm{DMU}_{\mathrm{k}}$ is under evaluation.

$$
\begin{array}{ll}
\theta^{*}(l, k)= & \min _{\lambda_{i}, \theta(l, k)} \theta(l, k) \\
\text { s.t. } \quad & \sum_{i \in F\left(J^{l}\right)} \lambda_{i} x_{p i} \leq \theta(l, k) x_{p k} \\
& \sum_{i \in F\left(J^{l}\right)} \lambda_{i} y_{r i} \geq y_{r k} \\
& \lambda_{i} \geq 0, \quad i \in F\left(J^{l}\right)
\end{array}
$$

where $i \in F\left(J^{l}\right)$ means $D M U_{j} \in J^{l}$. If $l=1$, model (4) is the original DEA model, and $E^{l}$ consists of the benchmarks. The DMUs in set $E^{l}$ define the first layer of the frontier. Two sets of $E^{l}$ identified for two groups are applied as variable-benchmarks to evaluate the efficiency of all DMUs by model (3). If situation (iii) exists, model (4) needs to be resolved by setting $l=2$. By removing the first layer of frontiers (marked $G_{1(1)}$ and $G_{2(1)}$ in Figure 3), some DMUs within the set of $P P S_{1}^{1} \cap P P S_{2}^{1}$, are further identified as benchmarks to form the second layer of frontiers (marked $G_{1(2)}$ and $G_{2(2)}$ ). The new PPSs (here referred to as $P P S_{1}^{2}$ and $P P S_{2}^{2}$ ), dominated by $G_{1(2)}$ and $G_{2(2)}$, are the subset of $P P S_{1}^{1}$ and $P P S_{2}^{1}$, making a smaller intersection (notated as $P P S_{1}^{2} \cap P P S_{2}^{2}$ ) between them. The process needs to be performed from $l=1$ to $l$ $=\mathrm{L}$, where the $L^{\text {th }}$ layers of frontiers dominate two subsets of PPSs having no intersection between them $\left(P P S_{1}^{L} \cap P P S_{2}^{L} \in \phi\right)$. Then, these two $L^{\text {th }}$ layers of frontiers can be applied as discriminant hyperplanes for classification.

The stratification DEA model mentioned above is performed based on a symmetric layering technique that is suitable for an even size of both groups $[17,18]$. For the bankruptcy prediction, removing a layer of the frontier from the non-bankrupt group will raise the risk of Type I error because of the reduced PPS range. If the frontier being removed is from the bankrupt group, it will increase the risk of Type II error. Generally, the cost of Type II error is much more expensive than the Type I error. To minimize the total cost of misclassification, an asymmetric layering technique is performed by incorporating error costs and risk rates into the expected cost of the misclassification (ECM) function to identify a pair of frontiers that can minimize the misclassification cost. That is:

$$
\min \quad E C M=c(2 \mid 1) P(2 \mid 1) p_{1}+c(1 \mid 2) P(1 \mid 2) p_{2}
$$




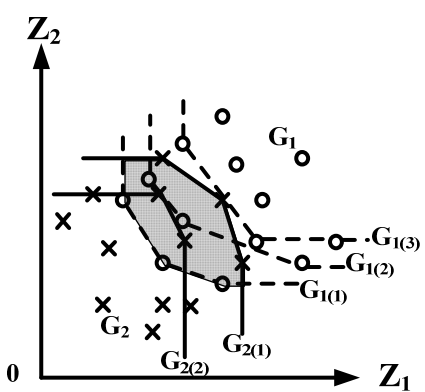

Figure 3. Two feasibilities of separating hyperplanes $\left(G_{1(2)}-\right.$ $G_{2(2)}$ and $G_{1(3)}-G_{2(1)}$.

where $c(2 \mid 1)$ is the cost of Type I error and $c(1 \mid 2)$ means the cost of Type II error. $P(2 \mid 1)$ and $P(1 \mid 2)$ are the risk rates of Type I and Type II errors, calculated with the accumulative number of benchmarks on the removed frontiers, divided by the total amount of DMUs in the corresponding group. The more frontiers removed from the PPSs, the greater the risk of Type I or Type II errors. The ratios of $p_{1}$ and $p_{2}$ are the proportions of non-bankrupt and bankrupt companies. Several combinations of the two sets of benchmarks (notated as $E_{1}^{*}$ and $E_{2}^{*}$ ) can dominate two PPSs having no intersection between them $\left(P P S_{1}^{L_{1}} \cap P P S_{2}^{L_{2}} \in \phi, L_{1} \neq L_{2}\right)$. All have various layers of removed frontiers on the two PPSs, which cause different risks of $P(2 \mid 1)$ and $P(1 \mid 2)$. An optimal set of $E_{1}^{*}$ and $E_{2}^{*}$ is obtained by solving model (5) to minimize the expected cost of misclassification. Then, the frontiers constructed by $E_{1}^{*}$ and $E_{2}^{*}$ are applied as discriminant hyperplanes, used to predict a new observation by the variable-benchmark DEA model (2). The rules of classification are:

$$
\begin{aligned}
& \text { If (i) } \delta_{G_{1}}^{*} \leq 1, \delta_{G_{2}}^{*}>1 \text {, then } D M U^{\text {new }} \in G_{1} \text {, } \\
& \text { (ii) } \delta_{G_{1}}^{*}>1, \delta_{G_{2}}^{*} \leq 1 \text {, then } D M U^{\text {new }} \in G_{2} \text {, } \\
& \text { (iii) } \delta_{G_{1}}^{*}>1, \delta_{G_{2}}^{*}>1 \text {, and } \delta_{G_{1}}^{*}<\delta_{G_{2}}^{*} \text {, then } D M U^{\text {new }} \in G_{1} \text {, } \\
& \text { (iv) } \delta_{G_{1}}^{*}>1, \delta_{G_{2}}^{*}>1 \text {, and } \delta_{G_{1}}^{*}>\delta_{G_{2}}^{*} \text {, then } D M U^{\text {new }} \in G_{2} \text {. }
\end{aligned}
$$

\section{Application}

A data set containing annual financial data was collected from Taiwan Stock Exchange for both failed and healthy companies in 2006 and 2007. Nineteen and eleven failed companies were matched with 160 and 115 healthy companies in the two years to present an uneven group size of the classification problem.

This study considered the 2006 data as training samples for model development and the 2007 data as holdout samples for validation purposes. This article examined the variables of total assets (TA), earnings before income, tax, depreciation and amortization (EBITDA), total current liabilities (CL), interest expense (IN), and cash flow from operations (CF) to be different significantly between bankrupt and non-bankrupt groups and selected them as discriminant factors.

To identify the worst performers to establish the boundary for the non-bankrupt group, those DUMs with the highest output of total assets (TA), total current liabilities (CL), and interest expense (IN) while having the lowest input level of earnings before income, tax, depreciation and amortization (EBITDA) and cash flow (CF), were identified as benchmarks. The same variables were used by define TA, CL and IN as input variables, and EBITDA and CF as output for the bankrupt group to identify the best performers. Because negative value is typical for some financial variables, to satisfy the positive restriction in the DEA model, any one of the selected factors taking on a negative value needs to add an adequate positive constant value to the factor value of all DMUs with the absolute value of the most negative value among those DMUs plus one. Two sets of benchmarks identified by the DEA model (1) with the aforementioned variables were applied to evaluate all DMUs by the variable-benchmark DEA model (2). The results indicated the existence of an overlap, because there were 64 DMUs dominated simultaneously by the first layers of the two groups, eight DMUs from bankruptcy, and 56 from non-bankruptcy. To eliminate the intersection, sequential layers of frontier were generated for non-bankrupt and bankrupt groups by performing the stratification DEA model (3). Tables 1 and $\mathbf{2}$ show the number of identified benchmarks in each layer of frontier and the risk occurring from the removed frontiers.

Because the sample of non-bankrupt and bankrupt companies is uneven, the number of layers of the removed frontiers is unequal. Tables $\mathbf{1}$ and $\mathbf{2}$ show that removing nine layers of frontier can move $100 \%$ of failed companies out of the PPS, while only $30 \%$ of the healthy companies were removed from the non-bankruptcy group. It is significant that the risks caused by the removed frontiers differ between the two groups. Removing a layer of frontier from the bankrupt group creates much more risk (Type II errors) than from the non-bankruptcy group (Type I errors), because the first layer of removed frontier causes only $1.25 \%$ risk in non- bankruptcy (Type I errors) but $10.53 \%$ in bankruptcy (Type II errors). Eight collocations of pair frontiers can make no intersection between two PPSs of non-bankrupt and bankrupt groups. To identify the optimal pair of frontiers to be separating hyperplanes, formula (5) is solved in accordance with different error risks and costs caused from each collocation to minimize expected cost of misclassification.

There are eight collocations of pair frontiers making no intersection between two PPSs of non-bankrupt and bankrupt groups. To identify the optimal pair of frontiers to be separating hyperplanes, formula (5) is solved in 
Table 1. The number of identified benchmarks on each frontier and the risk occurred from the removed frontiers of nonbankrupt group.

\begin{tabular}{|c|c|c|c|c|c|c|c|}
\hline Layer & $\begin{array}{l}\text { Number of } \\
\text { Benchmarks }\end{array}$ & $\begin{array}{c}\text { Accumulative } \\
\text { Number of Removed } \\
\text { Benchmarks }\end{array}$ & $\begin{array}{c}\text { Accumulative } \\
\text { Risk of Error } \\
P(2 \mid 1) \quad \%\end{array}$ & Layer & $\begin{array}{l}\text { Number of } \\
\text { Benchmarks }\end{array}$ & $\begin{array}{c}\text { Accumulative } \\
\text { Number of Removed } \\
\text { Benchmarks }\end{array}$ & $\begin{array}{c}\text { Accumulative } \\
\text { Risk of Error } \\
P(2 \mid 1) \%\end{array}$ \\
\hline 1 & 2 & 0 & 0.00 & 12 & 7 & 63 & 39.38 \\
\hline 2 & 3 & 2 & 1.25 & 13 & 8 & 70 & 43.75 \\
\hline 3 & 4 & 5 & 3.13 & 14 & 11 & 78 & 48.75 \\
\hline 4 & 9 & 9 & 5.63 & 15 & 7 & 89 & 55.63 \\
\hline 5 & 5 & 18 & 11.25 & 16 & 6 & 96 & 60.00 \\
\hline 6 & 4 & 23 & 14.38 & 17 & 8 & 102 & 63.75 \\
\hline 7 & 6 & 27 & 16.88 & 18 & 6 & 110 & 68.75 \\
\hline 8 & 8 & 33 & 20.63 & 19 & 9 & 116 & 72.50 \\
\hline 9 & 7 & 41 & 30.00 & 20 & 5 & 125 & 78.13 \\
\hline 10 & 10 & 48 & 36.25 & 21 & 4 & 130 & 81.25 \\
\hline 11 & 5 & 58 & 39.38 & & & 134 & 83.75 \\
\hline
\end{tabular}

Table 2. The number of identified benchmarks on each frontier and the risk occurred from the removed frontiers of bankrupt group.

\begin{tabular}{cccccccc}
\hline Layer & $\begin{array}{c}\text { Number of } \\
\text { Benchmarks }\end{array}$ & $\begin{array}{c}\text { Accumulative } \\
\text { Number of Removed } \\
\text { Benchmarks }\end{array}$ & $\begin{array}{c}\text { Accumulative } \\
\text { Risk of Error } \\
P(1 \mid 2) \%\end{array}$ & Layer & $\begin{array}{c}\text { Number of } \\
\text { Benchmarks }\end{array}$ & $\begin{array}{c}\text { Accumulative } \\
\text { Number of Removed } \\
\text { Benchmarks }\end{array}$ & $\begin{array}{c}\text { Accumulative } \\
\text { Risk of Error } \\
P(1 \mid 2)\end{array}$ \\
\hline 1 & 2 & 0 & 0.00 & 6 & 1 & 13 & 68.42 \\
2 & 2 & 2 & 10.53 & 7 & 1 & 14 & 73.68 \\
3 & 3 & 4 & 21.05 & 8 & 2 & 15 & 78.95 \\
4 & 3 & 7 & 36.84 & 9 & 2 & 19 & 89.47 \\
5 & 3 & 10 & 52.63 & & & 19 & 100.00 \\
\hline
\end{tabular}

accordance with different error risks and costs caused from each collocation to minimize expected cost of misclassification. The ratio of Type II cost to Type I cost $(c(1 \mid 2) / c(2 \mid 1))$ is assumed from 1 to 1 to 20 to 1 for the situation of different applications. Table 3 shows NB-2 and B-7 are the best choice if ignoring the influence of error cost (that is, assume $c(1 \mid 2) / c(2 \mid 1)$ equal to 1 ). For which, only $1.25 \%$ of healthy companies (the benchmarks on the first layer of frontier, see Table 1) are removed from the non-bankrupt group, but $78.95 \%$ of failed companies (the benchmarks removed from layer one to layer six, see Table 2) need to be removed from the bankrupt group because the classification accuracy of the smaller group is sacrificed to increase the hit-ratio. If Type II cost is much higher than Type I cost (as Table 3 shows, 20 times to Type I cost), the pair frontiers of NB-21 and B-1 is the best selection for the purpose of minimizing expected misclassification cost. Besides, if the pair of frontiers is determined by the symmetric- stratified DEA model, that is, remove the same number of layers from both groups, the misclassification cost is almost higher than the lowest value of all collocations determined by the asymmetric-stratified model. In accordance with the empirical studies of Altman (1993) and Hull (1998), the cost of Type II errors is in the range of 0.6 to 0.7 and Type I errors are derived from the interest spread of usually 3\% - 5\%. Therefore, this article assumed that Type II cost is 20 times to Type I cost, and the pair frontiers of NB-21 and B-1 were then the best selection to be discrimination hyperplanes for further analysis and discussion.

Table 4 shows the hit-ratios of training and holdout data. If the purpose is to maximize the hit-ratio, the frontiers of NB-2 and B-7 are the best choice with the hit-ratios of $94.44 \%$ on training and $91.67 \%$ on hold out samples. High prediction accuracy supports the validity of the proposed DEA approach, a result consistent with some traditional discriminant methods whose analyses 
Table 3. The cost of misclassification for different collocations of pair frontiers.

\begin{tabular}{cccccccccc}
\hline $\begin{array}{c}\text { Layer } \\
\text { Alternative }\end{array}$ & $\begin{array}{c}\text { NB-21 } \\
\text { B-1 } \\
\text { Cost Ratio }\end{array}$ & $\begin{array}{c}\text { NB-17 } \\
\text { B-2 } \\
(2)\end{array}$ & $\begin{array}{c}\text { NB-13 } \\
\text { B-3 } \\
(3)\end{array}$ & $\begin{array}{c}\text { NB-11 } \\
\text { B-4 } \\
(4)\end{array}$ & $\begin{array}{c}\text { NB-9 } \\
\text { B-5 } \\
(5)\end{array}$ & $\begin{array}{c}\text { NB-5 } \\
\text { B6 } \\
(6)\end{array}$ & $\begin{array}{c}\text { NB-2 } \\
\text { B-7 } \\
(7)\end{array}$ & $\begin{array}{c}\text { NB-1 } \\
\text { B-8 } \\
(8)\end{array}$ & $\begin{array}{c}\text { NB-6 } \\
\text { B-6 } \\
\text { symmetric }\end{array}$ \\
\hline$(c(1 \mid 2) / c(2 \mid 1))=1$ & 0.6806 & 0.5810 & 0.4134 & 0.3631 & 0.2850 & 0.1732 & $0.0838^{*}$ & 0.0894 & 0.2011 \\
$(c(1 \mid 2) / c(2 \mid 1))=5$ & 0.4712 & 0.4392 & 0.3529 & 0.3647 & 0.3569 & 0.3255 & $0.2823^{*}$ & 0.2941 & 0.3451 \\
$(c(1 \mid 2) / c(2 \mid 1))=10$ & 0.3403 & 0.3485 & $0.3143^{*}$ & 0.3657 & 0.4029 & 0.4229 & 0.4057 & 0.4286 & 0.4371 \\
$(c(1 \mid 2) / c(2 \mid 1))=15$ & $0.2663^{*}$ & 0.2966 & 0.2920 & 0.3663 & 0.4292 & 0.4786 & 0.4764 & 0.5056 & 0.4899 \\
$(c(1 \mid 2) / c(2 \mid 1))=20$ & $0.2118^{*}$ & 0.2629 & 0.2778 & 0.3667 & 0.4463 & 0.5148 & 0.5222 & 0.5556 & 0.5241 \\
\hline
\end{tabular}

Note: NB: Non-Bankruptcy, B: Bankruptcy, NB-21: The twenty-first layer of frontier in Non-Bankrupt group.

Table 4. The hit-ratio for training and holdout data and expected cost of misclassification for holdout data.

\begin{tabular}{|c|c|c|c|c|c|}
\hline $\begin{array}{c}\text { Layer } \\
\text { Hit-ratio } \\
\text { (holdout/training) } \\
\text { Cost Ratio }\end{array}$ & $\begin{array}{c}\text { NB-21, B-1 } \\
31.94 \% \\
34.92 \%\end{array}$ & $\begin{array}{c}\text { NB-13, B-3 } \\
58.33 \% \\
60.32 \%\end{array}$ & $\begin{array}{c}\text { NB-2, B-7 } \\
91.67 \% \\
94.44 \%\end{array}$ & $\begin{array}{c}\text { NB-1, B-8 } \\
90.28 \% \\
92.86 \%\end{array}$ & $\begin{array}{c}\text { NB-6, B-6 } \\
83.33 \% \\
84.92 \%\end{array}$ \\
\hline$(c(1 \mid 2) / c(2 \mid 1))=1$ & 0.6508 & 0.3968 & $0.0556^{*}$ & 0.0714 & 0.1508 \\
\hline$(c(1 \mid 2) / c(2 \mid 1))=5$ & 0.4824 & 0.3882 & $0.2059^{*}$ & 0.2647 & 0.2529 \\
\hline$(c(1 \mid 2) / c(2 \mid 1))=10$ & 0.3644 & 0.3822 & $0.3111^{*}$ & 0.4000 & 0.3244 \\
\hline$(c(1 \mid 2) / c(2 \mid 1))=15$ & $0.2929^{*}$ & 0.3786 & 0.3750 & 0.4821 & 0.3679 \\
\hline$(c(1 \mid 2) / c(2 \mid 1))=20$ & $0.2448^{*}$ & 0.3761 & 0.4179 & 0.5373 & 0.3970 \\
\hline
\end{tabular}

are based on the assumption of equal cost of Type I and Type II errors. Table 4 shows the hit-ratio and misclassification cost tradeoff if the proportion of Type II to Type I cost is greater than ten, that is, a higher hit-ratio will be accompanied with a greater misclassification cost. Figure 4 shows that in the case of equal cost of Type I and Type II errors, a $94.44 \%$ hit-ratio can result in a lower misclassification cost among other hit-ratios, but in an uneven cost of Type I and Type II errors, the higher hit-ratio will result in a higher misclassification cost.

\section{Conclusion}

This article introduces an asymmetric-stratified DEA approach, which establishes nonlinear discriminant functions by the benchmarks of non-bankrupt and bankrupt groups. Instead of evaluating the prediction capability by the hit-ratio that tends to have upward bias towards the larger group (non-bankruptcy) to improve discrimination performance, the proposed approach establishes discriminant functions by minimizing the total expected misclassification cost (EMC). The aforementioned results indicate that our approach can perform high classification and prediction accuracy with hit-ratios of $94.44 \%$ on training and $91.67 \%$ on hold-out samples, but it is effective only if the cost of Type II error is equal or close to that of Type I error. If the proportion of Type II

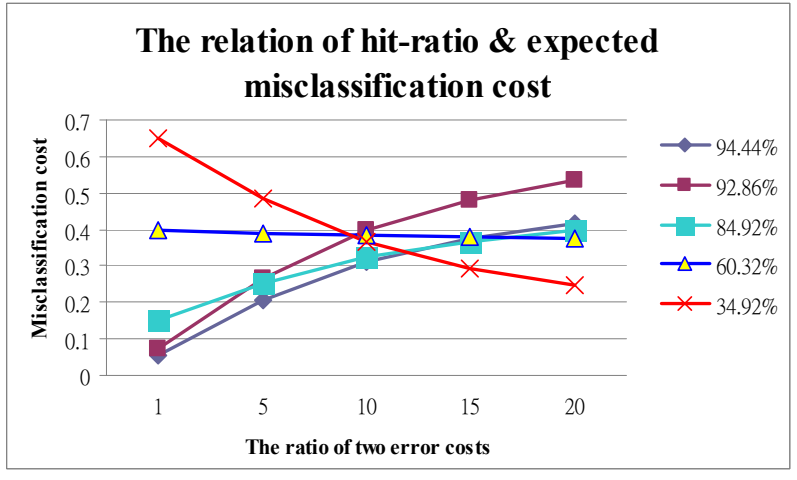

Figure 4. Relation of hit-ratio\& expected misclassification cost.

to Type I cost is greater than ten, a tradeoff occurs between hitratio and misclassification cost, meaning that a higher hit-ratio is accompanied with greater misclassification cost. When the ratio of two error costs is assumed 20 to 1, the separating hyperplanes of frontiers NB-21 and B-1 determine the best solution to prevent the appearance of Type II error to minimize expected misclassification cost, even though the hit-ratio of training data is only $34.92 \%$ and holdout is $31.94 \%$. Therefore, a highest hit-ratio is not an absolute best measurement for all situations of classification problems. By incorporating different errors cost and risks into the procedure, the 
proposed benchmark approach can easily establish more than one separating hyperplane and provide flexibility to decision makers for credit evaluation and bankruptcy prediction.

\section{REFERENCES}

[1] E. I. Altman, "Financial Ratios, Discriminant Analysis and the Prediction of Corporate Bankruptcy,” The Journal of Finance, Vol. 23, No. 4, 1968, pp. 589-609. http://dx.doi.org/10.1111/j.1540-6261.1968.tb00843.x

[2] A. I. Dimitras, C. Zopounidis and C. Hurson, "A Multicriteria Decision Aid Method for the Assessment of Business Failure Risk," Foundations of Computing and Decision Sciences, Vol. 20, No. 2, 1995, pp. 99-112.

[3] A. I. Dimitras, S. H. Zanakis and C. Zopounidis, “A Survey of Business Failures with an Emphasis on Prediction Methods and Industrial Applications,” European Journal of Operation Research, Vol. 90, No. 3, 1996, pp. 487-513. http://dx.doi.org/10.1016/0377-2217(95)00070-4

[4] B. E. Deakin, "A Discriminant Analysis of Predictors of Business Failure,” Journal of Accounting Research Spring, Vol. 10, No. 1, 1976, pp.167-179.

[5] M. D. Troutt, A. Rai and A. Zhang, "The Potential Use of DEA for Credit Applicant Acceptance Systems,” Computers Operations Research, Vol. 23, No. 4, 1996, pp. 405-408.

http://dx.doi.org/10.1016/0305-0548(95)00048-8

[6] D. Retzlaff-Roberts and R. Puelz, "Classification in Automobile Insurance Using a DEA and Discriminant Analysis Hybrid," Journal of Productivity Analysis, Vol. 7, No. 4, 1996, pp. 417-427. http://dx.doi.org/10.1007/BF00162050

[7] L. M. Seiford and J. Zhu, “An Acceptance System Decision Rule with Data Envelopment Analysis,” Computers Operations Research, Vol. 25, No. 4, 1998, pp. 329-332. http://dx.doi.org/10.1016/S0305-0548(97)00075-0

[8] P. C. Pendharkar, "A Potential Use of Data Envelopment Analysis for the Inverse Classification Problem,” Omega, Vol. 30, No. 3, 2002, pp. 243-248.

http://dx.doi.org/10.1016/S0305-0483(02)00030-0

[9] A. Cielen, L. Peeters and K. Vanhoof, "Bankruptcy Prediction Using a Data Envelopment Analysis," European Journal of Operational Research, Vol. 154, No. 2, 2004, pp. 526-532. http://dx.doi.org/10.1016/S0377-2217(03)00186-3

[10] J. C. Paradi, M. Asmild and P. C. Simak, "Using DEA and Worst Practice DEA in Credit Risk Evaluation,” Journal of Productivity Analysis, Vol. 21, No. 2, 2004, pp. 153-165.

http://dx.doi.org/10.1023/B:PROD.0000016870.47060.0b

[11] T. Sueyoshi and M. Goto, "DEA-Discriminant Analysis: Methodological Comparison Among Eight Discriminant Analysis Approaches,” European Journal of Operational Research, Vol. 169, No. 1, 2006, pp. 247-272. http://dx.doi.org/10.1016/j.ejor.2004.05.025

[12] T. Sueyoshi and M. Goto, "DEA-DA for BankruptcyBased Performance Assessment: Misclassification Analysis of Japanese Construction Industry,” European Journal of Operational Research, Vol. 199, No. 2, 2009, pp. 576594. http://dx.doi.org/10.1016/j.ejor.2008.11.039

[13] M. Psillaki, I. E. Tsolas and D. Margaritis, "Evaluation of Credit Risk Based on Firm Performance," European Journal of Operational Research, Vol. 201, No. 3, 2010, pp. 873-881. http://dx.doi.org/10.1016/j.ejor.2009.03.032

[14] A. Charnes and W. W. Cooper, "Goal Programming and Multiple Objective Optimization,” European Journal of Operational Research, Vol. 1, No. 1, 1977, pp. 39-54. http://dx.doi.org/10.1016/S0377-2217(77)81007-2

[15] A. Charnes, W. W. Cooper and E. Rhode, "Measuring the Efficiency of Decision Making Units,” European Journal of Operational Research, Vol. 2, No. 6, 1978, pp. 429444. http://dx.doi.org/10.1016/0377-2217(78)90138-8

[16] J. Zhu, “Quantitative Models for Performance Evaluation and Benchmarking: Data Envelopment Analysis with Spreadsheets and DEA Excel Solver,” Kluwer Academic Publishers, Boston, 2003. http://dx.doi.org/10.1007/978-1-4757-4246-6

[17] D. S. Chang and Y. I. Kuo, “A Novel Procedure to Identify the Minimized Overlap Boundary of Two Groups by DEA Model,” Lecture Notes in Computer Science, Vol. 3483, 2005, pp. 577-586. http://dx.doi.org/10.1007/11424925_61

[18] D. S. Chang and Y. I. Kuo, "An Approach for the TwoGroup Discriminant Analysis: An Application of DEA,” Mathematic \& Computer Modelling, Vol. 47, No. 9-10, 2008, pp. 970-981. http://dx.doi.org/10.1016/j.mcm.2007.05.010 